\title{
APACHE II Death Risk and Length of Stay in the ICU Are Associated With Pressure Injury in Critically III Patients
}

\author{
Francine Sanchez Gulin ${ }^{\mathrm{a}, \mathrm{b}}$, Mayra Goncalves Menegueti $\mathrm{a}$, b, d, Maria Auxiliadora-Martins ${ }^{\mathrm{b}}$, \\ Thamiris Ricci de Araujo ${ }^{a}$, Fernando Bellissimo-Rodrigues ${ }^{c}$, Aline Nassiff ${ }^{a}$, \\ Anibal Basile-Filho ${ }^{\mathrm{b}}$, Ana Maria Laus ${ }^{\mathrm{a}}$
}

\begin{abstract}
Background: The aim of this study was to identify factors associated with pressure injury (PI) occurrence in critically ill patients. This was a retrospective cohort study conducted at a mixed intensive care unit (ICU).

Methods: Univariate analysis and logistic regression were used to identify which variables are associated with PI.

Results: Twenty-one (15\%) of 142 patients developed PI. The median and the range of the variables in the groups without and with PI were as follows: Braden scale risk score, $13(8-20)$ and $10(8$ - 14) points, respectively; Acute Physiology and Chronic Health Evaluation II (APACHE II) death risk, 39\% (2 - 97\%) and 75\% (26 - 96\%), respectively; and length of stay in the ICU, 4 (2 - 36) and 16 (5 - 29) days, respectively. The socio-demographic variables included in the logistic regression were age $(\mathrm{P}=0.09)$, Braden scale risk score $(\mathrm{P}=$ $0.0003)$, APACHE II death risk ( $\mathrm{P}<0.0001)$, length of stay in the ICU $(\mathrm{P}<0.0001)$ and reason for ICU admission $(\mathrm{P}=0.09)$. Only APACHE II death risk and length of stay in the ICU presented significant differences; the odds ratios were 1.05 and 1.25 , respectively, and the $95 \%$ confidence intervals were $1.01-1.09$ and $1.12-1.39$, respectively.
\end{abstract}

Conclusion: APACHE II death risk and length of stay in the ICU are probably associated with PI occurrence in the study population.

Keywords: APACHE II death risk; Critical care; Critical illness;

Manuscript submitted September 22, 2018, accepted October 16, 2018

aDepartment of Fundamental Nursing, University of Sao Paulo at Ribeirao Preto College of Nursing, Ribeirao Preto, Sao Paulo, Brazil

${ }^{b}$ Department of Surgery and Anatomy, Division of Intensive Care University of Sao Paulo at Ribeirao Preto Medical School, Ribeirao Preto, Sao Paulo, Brazil

'Department of Social Medicine, University of Sao Paulo at Ribeirao Preto Medical School, Ribeirao Preto, Sao Paulo, Brazil

${ }^{\mathrm{d} C}$ Corresponding Author: Mayra Goncalves Menegueti, Ribeirao Preto College of Nursing, University of Sao Paulo (EERP/USP), Av. Bandeirantes, s/n - Bairro Monte Alegre, Ribeirao Preto, SP CEP 14049-900, Brazil.

Email: mayramenegueti@usp.br

doi: https://doi.org/10.14740/jocmr3636
Pressure injury

\section{Introduction}

Healthcare quality and patient safety are matters of great concern to hospital systems: if any damage or lesion attributable to the delivered care occurs, there will certainly be undesirable consequences for both the patient and the health institution [1]. Adverse effects are an indicator of healthcare quality and reflect the delivered care. Mitigating the occurrence of such effects makes healthcare safer [2]. One of the relevant aspects of critical patient care refers to skin integrity maintenance. Pressure injury (PI) emerges at all healthcare levels and is particularly common in patients with reduced mobility and older age, which makes PI a clinical care indicator and one of the most frequent adverse effects in critically ill patients.

Over the last decade, studies involving critical patients have pointed to PI incidence ranging from $3.3 \%$ to $39 \%[3,4]$. A literature review of European studies spanning from 2000 to 2005 identified that the PI incidence varied from $3.8 \%$ to $12.4 \%$ [5]. An investigation conducted in China verified a PI incidence of $0.63 \%$; the intensive care unit (ICU) was the sector with the highest coefficient [6]. In Brazil, the PI incidence lies between $10 \%$ and $62.5 \%$ [7, 8]. Along patient recovery, high PI incidence could culminate in complications like higher risk of hospital-acquired infection and hospital malnutrition, longer hospital stay, increased nursing staff workload and higher healthcare costs.

Many factors have been reported to be associated with PI development, especially extrinsic pressure related to older age, nutritional alterations or deficit, moisture, immobility or bed rest, lower tissue perfusion, long hospital stay, and comorbidities such as diabetes mellitus, nephropathy and vascular disease, among other factors. In this context, critical patients are more exposed to PI [9-11].

The medical literature contains controversial results concerning the contribution of different risk factors to PI development, which depend on the patient's characteristics, comorbidities and healthcare scenarios. There are evidences that intrinsic and extrinsic factors predispose to PI development, so identifying risk factors during patient care is an asset to hospi- 


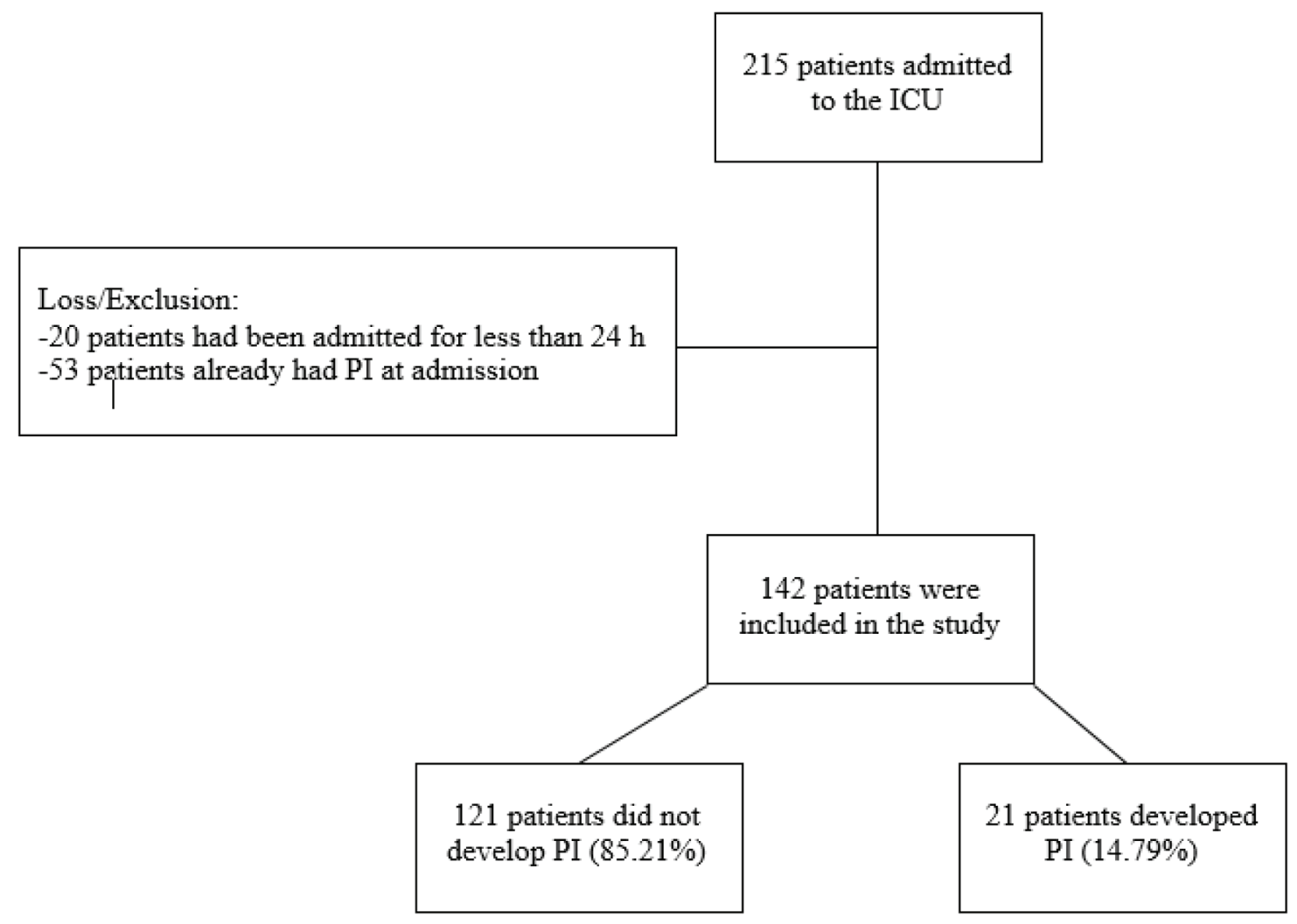

Figure 1. Study screening process.

tal institutions, which can plan specific intervention measures and put them into practice in order to prevent PI [9].

Preventing PI is one of the most important tasks conducted by nurses in ICUs. This study aims to identify which factors are associated with PI occurrence in critically ill patients.

\section{Patients and Methods}

This is a quantitative, descriptive, retrospective cohort study conducted at an ICU of a large high-complexity tertiary university hospital in the interior of the state of Sao Paulo. This study was approved by the research ethics committee (process number 7076).

This study included male and female patients aged at least 18 years and who were admitted to the hospital between January and June 2015 for clinical or surgical treatment. Inclusion criteria included first patient admission to the ICU, stay in the ICU for at least $24 \mathrm{~h}$ and absence of PI at the time of admission as evaluated by an ICU nurse. Figure 1 illustrates the inclusion flow chart.

Patients' socio-demographic and clinical data were obtained from the electronic medical records filed at the hospital. To characterize patient severity, the Acute Physiology and Chronic Health Evaluation II (APACHE II) death risk score was determined upon admission. In the case of critically ill patients, several scoring systems have been developed over the years. In the ICU setting, APACHE II is one of the most widely used scoring systems. This score, which ranges from 0 to 71 , considers 12 physiological variables as well as the Glasgow coma scale (calculated after the effect of anesthetic agents is abolished), age, need for urgent surgical procedures and comorbidities verified in the patient's medical records. The higher the APACHE II score, the greater the patient's death risk [12].

The Braden scale risk scores, which are used to measure the risk of PI, were collected from an assessment instrument that was filled in by the ICU nurse at the time of admission. Score 18 of said scale was adopted as cutoff point for PI risk as recommended in the literature. The patients were classified as very high risk (scores equal to or lower than 9), high risk (scores between 10 and 12 points), moderate risk (scores between 13 and 14 points), low risk (scores between 15 and 18 points) and no risk (scores between 19 and 23 points) [13].

\section{Statistical analysis}

The statistical analyses were performed with the program Stata SE® version 14.0 (Stata Corp., College Station, TX, USA). Data are reported as the median ( $\min$ - $\max$ ). Univariate analysis was accomplished. Fisher's exact test was applied for the qualitative variables, and the Mann-Whitney/Wilcoxon test was applied to the quantitative variables because they did not follow a normal distribution as revealed by the KolmogorovSmirnov test. Variables of age in years, Braden scale risk 
Table 1. Qualitative Variables in Patients Without and With Pressure Injury (PI) and Univariate Analysis

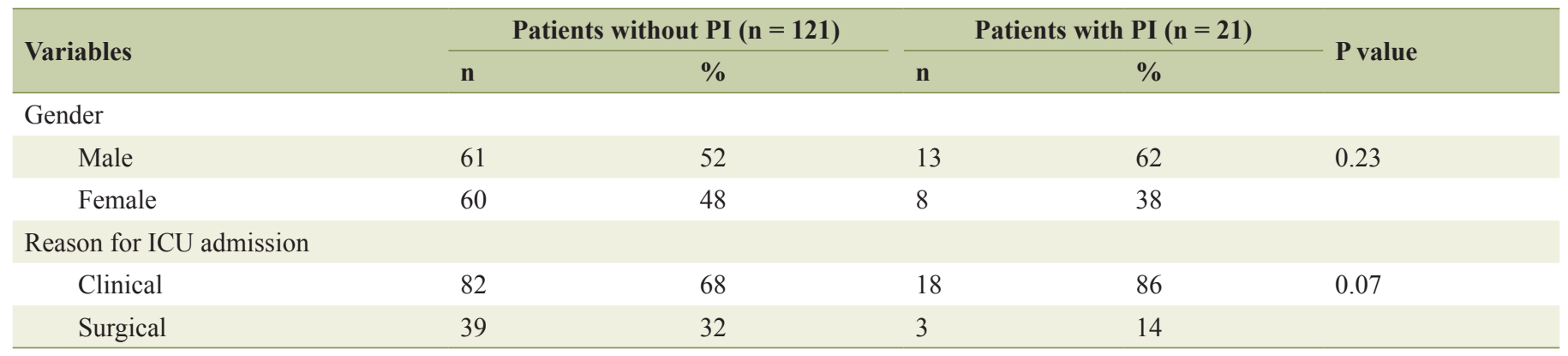

PI: pressure injury; P value: univariate analysis by Fisher's exact test.

Table 2. Quantitative Variables in Patients Without and With Pressure Injury (PI) and Univariate Analysis

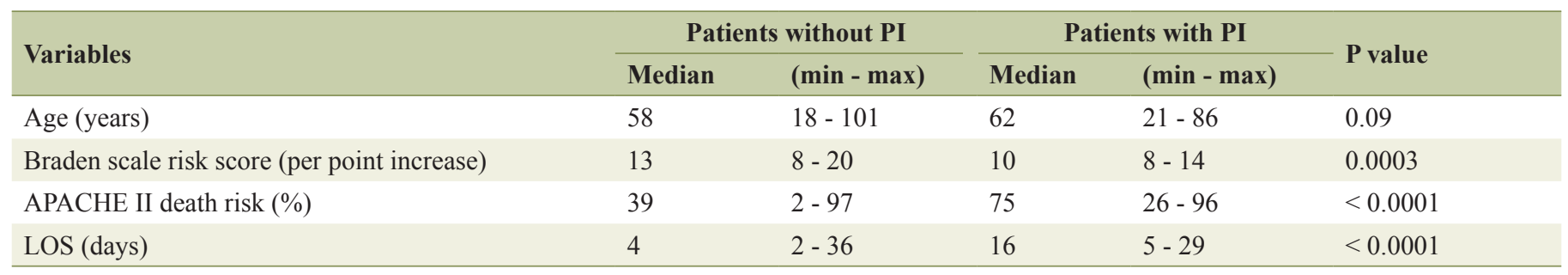

LOS: length of stay in the ICU; PI: pressure injury; P value: univariate analysis by Wilcoxon test.

score, APACHE II death risk (\%), length of stay in the ICU in days and reason for ICU admission were inserted in the logistic regression model because that presented at $\mathrm{P}<0.20$ in the univariate analysis. The level of significance $(\alpha)$ was set at $5 \%$, with $\mathrm{P}$ value $<0.05$.

\section{Results}

During the study period, 142 patients met the inclusion criteria. Among these patients, 21 developed PI, which corresponded to an incidence of $14.79 \%$ (Fig. 1). Table 1 lists the qualitative variables.

The group without PI contained practically the same number of female and male patients. Male patients prevailed in the group with PI, albeit without significance.

Compared to clinical patients, surgical patients predominated in both groups (without and with PI). Although there was no statistical difference, this variable was included in the final model because $\mathrm{P}=0.07$

Table 2 summarizes the quantitative variables.

As for age, the median age in the group without PI was lower than the median age in the group with PI. Despite the absence of statistical difference, this variable was included in the multivariate analysis because $\mathrm{P}=0.09$. The median Braden scale risk score for PI evidenced moderate and high risk in the groups without and with PI, respectively, with statistical significance. The median APACHE II death risk was higher in the group with PI (75\%) as compared to the group without PI $(39 \%)$, also with statistical significance. The median length of stay in the ICU was much higher in the group with PI (16 days) as compared to the group without PI (4 days).

The variables included in the logistic regression were age in years, Braden scale risk score, APACHE II death risk (\%), length of stay in the ICU in days and reason for ICU admission (Table 3).

In the final model, APACHE II death risk (\%) and length of stay in the ICU were associated with PI occurrence.

Table 3. Multivariate Analysis of Pressure Injury (PI) Considering Age, Braden Scale Risk Score, Reason for ICU Admission, APACHE II Death Risk and Length of Stay in the ICU

\begin{tabular}{llll}
\hline Variable & Odds ratio (OR) & Confidence interval (95\% CI) & P value \\
\hline Age & 1.02 & $0.98-1.06$ & 0.34 \\
Braden scale risk score (per point increase) & 0.84 & $0.61-1.14$ & 0.26 \\
Reason for ICU admission & 0.22 & $0.03-1.79$ & 0.16 \\
APACHE II death risk (\%) & 1.05 & $1.01-1.09$ & 0.008 \\
LOS (days) & 1.25 & $1.12-1.39$ & 0.000 \\
\hline
\end{tabular}

LOS: length of stay in the ICU. 


\section{Discussion}

Programs for PI prevention based on international guidelines have impacted PI occurrence. However, not all the risk factors for PI development related to patient's characteristics, associated illness and healthcare level are known. Actually, there is no single factor accounting for PI occurrence. In fact, the interaction of many factors increases the probability of PI development [14].

In the literature, there is no consensus on whether the variable gender is related to PI etiology. Nevertheless, our results corroborate the results of other studies and show that gender is not associated with PI occurrence and should therefore be presented as a demographic characteristic only [9, 15].

Our study has not found any association between the variable age and PI development, either. An investigation conducted in 22 ICUs of 15 public and private hospitals in the state of Minas Gerais achieved the same result, but elderly patients were at higher risk of PI occurrence [16].

In another investigation into patients admitted to an intensive and semi-intensive care unit in the state of Rio Grande do Sul, researchers verified that patients who developed PI were significantly older than patients without PI (63.2 \pm 16.9 years versus $54.9 \pm 16.8$ years, respectively; $\mathrm{P}=0.018$ ) [11].

Although age as an associated factor in PI occurrence is controversial, it is worth highlighting that senescence implies changes in the integumentary tissue involving structural defects in the stratum corneum and in the epidermis, which decreases protection against external agents. In this sense, total body mass loss, which results in loose skin folds, increases the risk of integumentary trauma. Peripheral sensitivity alterations inherent to older age must also be considered [16]. Age is also relevant when it comes to the lower ability of the skin to resist mechanical load alone or in association with variables like illnesses, life style and length of stay in the ICU, which would justify the larger PI prevalence among patients older than 60 years [9].

The impact of the variable length of stay in the ICU as a factor for PI development has proven to be consistent. An investigation conducted in the ICUs of two large metropolitan hospitals in Saudi Arabia monitored 84 patients until they were discharged or until they died. The investigators reported mean length of stay in the ICU of 13.3 days for patients with PI, which constituted a predictor for all PI stages [17].

A tertiary university hospital in Germany was the setting for an investigation that followed patients admitted between 2007 and 2011. Univariate analysis helped to identify length of stay as one of the significant predictors for PI development. Risk for PI occurrence was higher in patients who stayed more than 30 days in the hospital [18].

Another study reported that PI emerged in patients that stayed for 9.5 days or longer in a surgical ICU. This was associated with reduced mobility, insertion of numerous devices in the skin and use of sedatives [9].

A study conducted in two ICUs (a surgical ICU and an infectious disease ICU) of a university hospital in Recife verified that length of stay higher than 10 days $(\mathrm{P}<0.001)$ was associated with PI development. The mean elapsed time between admission to the ICU and PI emergence was 6.9 days, but the authors of this study highlighted that they might have overestimated this time because skin inspection was not carried out on a daily basis [19].

The present study found that APACHE II death risk is associated with PI. This finding agrees with the findings of a research that investigated the relation between severity measured by APACHE II and PI development. The authors observed higher PI prevalence in more critically ill patients. However, when they applied the logistic regression model, this variable lost significance as a result of sample size or of low mean APACHE II score [20]. In a general ICU in Spain, researchers pointed out that APACHE II and simplified acute physiology score III (SAPS 3) could help to predict PI occurrence [21].

Researchers who used SAPS 3 verified that patients with PI were more critically ill $(\mathrm{P}=0.043)$, which reflected unstable hemodynamic conditions in patients taking vasoactive drugs, sedatives and painkillers [22].

Nonetheless, another study reached different conclusions - the mean APACHE II scores were similar in patients without and with PI $(\mathrm{P}=0.689)$. This could be attributed to the higher specificity of the investigated ICU, which assisted mainly heart surgery patients, who could present other more relevant variables for PI development [9].

Scores are crucial when assessing the risk for PI occurrence because they highlight vulnerable points and reinforce the importance of continuous assessment. Here, the Braden scale risk scores demonstrate moderate and high risk in the groups without and with PI, respectively. Only the univariate analysis shows statistical significance for this variable.

By means of univariate analysis, a literature study also verified significant difference between scores of critical patients without and with PI $(\mathrm{P}=0.001)$. Patients with PI had mean Braden scale risk score of 11 (variation between 8 and 19) $[8]$.

Other authors identified a median Braden scale risk score of 10 (high risk) for patients with PI (scores ranged from 6 to 19); $59 \%$ of the patients were at high risk. Patients without PI had a median Braden risk score of 14 (variation from 8 to 20); $68 \%$ of these patients had moderate risk of developing PI ( $\mathrm{P}=$ $0.003)$ [23].

Another work evaluated various wards of a large public university emergency hospital. Patients with lower Braden scale risk score developed PI. The PI point prevalence was $40 \%$. The intensive and semi-intensive care units had greater PI occurrence and lower Braden scale risk scores [24].

Although some researchers reinforce the association between risk for PI development and low Braden scale risk score verified in national and international studies, especially in critically ill patients, they also warn that it is necessary to pay attention to the subscales that constitute the scores when implementing skin care intervention measures [8, 24].

In contrast, another study identified the predictive validity of risk assessment scales at the same time that it emphasized the need to take the health professional's clinical experience and knowledge of the process into account [23].

When it comes to scales for critically ill patients' skin assessment, the Cubbin-Jackson scale risk score, designed in 1991, is worth highlighting. This score is specific for critical 
patients because it has better predictive value $(89 \%$ sensitivity and $61 \%$ specificity) as compared to the Braden scale risk score. Unfortunately, the Cubbin-Jackson scale risk score has not been validated in Brazil, so it is not available for use in this country [9].

The limitation of this study was the small number of patients with PI, which may interfere in the identification of possible risk factors for IP. We should also mention that the study was conducted in a single center.

\section{Implications for clinical practice}

Even though a single factor cannot explain PI occurrence, as mentioned above, and although there might be no direct or effective measure to be taken in order to prevent PI, in some cases interventions geared toward a single element can modify the effects of the other underlying factors.

Health institutions have sought to reduce undesirable situations originating from issues with patient care. In case these institutions cannot avoid the occurrence of adverse events, they take measures that limit damage and reduce the chances that this event will happen again. Recognizing PI as an adverse event related to patient safety represents a considerable progress in Brazil if we remember that PI has been internationally regarded as a serious public health concern for years.

Considering the critical status of patients admitted to the ICU, identifying which patients are at risk of developing PI allows the multi-professional team to define and to implement preventive measures with a collective responsibility mindset. In this sense, mitigating the impact of PI calls for knowledge of the factors associated with PI occurrence, so that the outcome is favorable.

\section{Conclusions}

Our results suggest that APACHE II death risk and length of stay in the ICU are associated with IP occurrence in the study population. Knowing which factors are associated with IP occurrence in critically ill patients will allow nursing leaders to develop continuing education programs to reduce the incidence of this severe complication.

\section{Conflict of Interest}

None of the authors has any conflict of interest to disclose.

\section{References}

1. Roque KE, Tonini T, Melo EC. Adverse events in the intensive care unit: impact on mortality and length of stay in a prospective study. Cad Saude Publica. 2016;32(10):e00081815.

2. Mendes W, Travassos C, Martins M, Marques PM. Adjustment of adverse events assessment forms for use in
Brazilian hospitals. Rev Bras Epidemiol. 2008;11:55-66.

3. Nassaji M, Askari Z, Ghorbani R. Cigarette smoking and risk of pressure ulcer in adult intensive care unit patients. Int J Nurs Pract. 2014;20(4):418-423.

4. Shahin ES, Dassen T, Halfens RJ. Incidence, prevention and treatment of pressure ulcers in intensive care patients: a longitudinal study. Int J Nurs Stud. 2009;46(4):413421.

5. Shahin ES, Dassen T, Halfens RJ. Pressure ulcer prevalence and incidence in intensive care patients: a literature review. Nurs Crit Care. 2008;13(2):71-79.

6. Jiang Q, Li X, Qu X, Liu Y, Zhang L, Su C, Guo X, et al. The incidence, risk factors and characteristics of pressure ulcers in hospitalized patients in China. Int J Clin Exp Pathol. 2014;7(5):2587-2594.

7. Diccini S, Camaduro C, Iida LIS. The incidence of pressure ulcer in neurosurgical patients from a university hospital. Acta Paul Enferm. 2009;22:205-209.

8. Fernandes LM, Caliri MH. Using the Braden and Glasgow scales to predict pressure ulcer risk in patients hospitalized at intensive care units. Rev Lat Am Enfermagem. 2008;16(6):973-978.

9. Campanili TC, Santos VL, Strazzieri-Pulido KC, Thomaz PB, Nogueira PC. Incidencia de ulceras por pressao em pacientes de Unidade de Terapia Intensiva Cardiopneumologica. Rev Esc Enferm USP. 2015;49:7-14.

10. Coqueiro JM, Brito RS. Multiple risk factors and preventive strategies of pressure ulcers: systematic review. Journal of Nursing UFPE Online. 2013;7 https://periodicos. ufpe.br/revistas/revistaenfermagem/article/view/12259

11. Rockenbach CWF, Borges AM, Amaral RB, Bordin E. Fatores de risco para desenvolvimento de ulceras de pressao em UTI. Conscientia e Saude. 2012;11. http://www. redalyc.org/articulo.oa?id=92923674007.

12. Knaus WA, Draper EA, Wagner DP, Zimmerman JE. APACHE II: a severity of disease classification system. Crit Care Med. 1985;13(10):818-829.

13. Ayello EA, Braden B. How and why to do pressure ulcer risk assessment. Adv Skin Wound Care. 2002;15(3):125131; quiz 132-133.

14. Lima Serrano M, Gonzalez Mendez MI, Carrasco Cebollero FM, Lima Rodriguez JS. Risk factors for pressure ulcer development in Intensive Care Units: A systematic review. Med Intensiva. 2017;41(6):339-346.

15. Smit I, Harrison L, Letzkus L, Quatrara B. What factors are associated with the development of pressure ulcers in a medical intensive care unit? Dimens Crit Care Nurs. 2016;35(1):37-41.

16. Gomes FS, Bastos MA, Matozinhos FP, Temponi HR, Velasquez-Melendez G. Factors associated to pressure ulcer in patients at adult Intensive Care Units. Rev Esc Enferm USP. 2010;44:1070-1076.

17. Tayyib N, Coyer F, Lewis P. Saudi Arabian adult intensive care unit pressure ulcer incidence and risk factors: a prospective cohort study. Int Wound J. 2016;13(5):912919.

18. Petzold T, Eberlein-Gonska M, Schmitt J. Which factors predict incident pressure ulcers in hospitalized patients? A prospective cohort study. Br J Dermatol. 
2014;170(6):1285-1290.

19. Oliveira Costa AC, Sabino Pinho CP, Almeida dos Santos AD, Santos do Nascimento AC. [Pressure ulcer: incidence and demographic, clinical and nutrition factors associated in intensive care unit patients]. Nutr Hosp. 2015;32(5):2242-2252.

20. Ulker Efteli E, Yapucu Gunes U. A prospective, descriptive study of risk factors related to pressure ulcer development among patients in intensive care units. Ostomy Wound Manage. 2013;59(7):22-27.

21. Catala Espinosa AI, Hidalgo Encinas Y, Cherednichenko T, Flores Lucas I, Gonzalez Tamayo R, Garcia-Martinez MA, Herrero-Gutierrez E. [Correlation between body mass index and development of pressure ulcers in intensive care medicine]. Enferm Intensiva. 2014;25(3):107-113.

22. Cremasco MF, Wenzel F, Sardinha FM, Zanei SSV, Whitaker IY. Pressure ulcer: patient risk, patient acuity, and nursing workload. Acta Paul Enferm. 2009;22:897-902.

23. Borghardt AT, Prado TN, Bicudo SDS, Castro DS, Bringuente MEO. Ulcera por pressao em pacientes criticos: incidencia e fatores associados. Rev Bras Enferm. 2016;69:460-467.

24. Bernardes RM, Caliri MHL. Pressure ulcer prevalence in emergency hospitals: a cross-sectional study. Online Braz J Nurs. 2016;15. http://www.objnursing.uff.br/index.php/ nursing/ article/view/5391. 